\title{
Self-healing in fractured GaAs nanowires
}

\author{
Jun Wang ${ }^{\mathrm{a}, \mathrm{b}}$, Chunsheng Lu ${ }^{\mathrm{b}, *}$, Qi Wang ${ }^{\mathrm{c}, *}$, Pan Xiao ${ }^{\mathrm{d}}$, Fujiu Ke ${ }^{\mathrm{a}, \mathrm{d}}$, Yilong Bai ${ }^{\mathrm{d}}$, \\ Yaogen Shen ${ }^{\mathrm{e}}$, Yanbo Wang ${ }^{\mathrm{f}}$, Bin Chen ${ }^{\mathrm{f}}$, Xiaozhou Liao ${ }^{\mathrm{f}}$, Huajian Gao ${ }^{\mathrm{g}}$ \\ ${ }^{a}$ School of Physics and Nuclear Energy Engineering, Beihang University, Beijing 100191, China \\ ${ }^{\mathrm{b}}$ Department of Mechanical Engineering, Curtin University, Perth, Western Australia 6845, Australia \\ ${ }^{\mathrm{c}}$ School of Aeronautics Science and Engineering, Beihang University, Beijing 100191, China \\ ${ }^{\mathrm{d}}$ State Key Laboratory of Nonlinear Mechanics (LNM), Institute of Mechanics, Chinese Academy of Sciences, Beijing 100190, China \\ ${ }^{\mathrm{e}}$ Department of Mechanical and Biomedical Engineering, City University of Hong Kong, Kowloon, Hong Kong, China \\ ${ }^{\mathrm{f}}$ School of Aerospace, Mechanical and Mechatronic Engineering, University of Sydney, Sydney, New South Wales 2006, Australia \\ ${ }^{\mathrm{g}}$ School of Engineering, Brown University, Providence, RI 02912, United States
}

Received 22 May 2012; received in revised form 3 July 2012; accepted 4 July 2012

Available online 14 August 2012

\begin{abstract}
Molecular dynamics simulations are performed to investigate a spontaneous self-healing process in fractured GaAs nanowires with a zinc blende structure. The results show that such self-healing can indeed occur via rebonding of Ga and As atoms across the fracture surfaces, but it can be strongly influenced by several factors, including wire size, number of healing cycles, temperature, fracture morphology, oriented attachment and atomic diffusion. For example, it is found that the self-healing capacity is reduced by $46 \%$ as the lateral dimension of the wire increases from 2.3 to $9.2 \mathrm{~nm}$, and by $64 \%$ after 24 repeated cycles of fracture and healing. Other factors influencing the self-healing behavior are also discussed.
\end{abstract}

(C) 2012 Acta Materialia Inc. Published by Elsevier Ltd. All rights reserved.

Keywords: GaAs nanowires; Self-healing; Size effect; Multiple healing; Molecular dynamics

\section{Introduction}

Self-healing refers to autonomous processes of restoring damaged materials to their original properties. Such behaviors are widely observed in natural materials, particularly in biomaterials, e.g. the healing of a small cut in skin and fractured bones. Mimicking biological systems, a number of synthetic materials have now been designed with the ability to spontaneously restore their mechanical properties and functions upon damage [1-5]. Over the last few years, self-healing has been observed in a wide range of nanostructured materials, such as ceramic nanocrystals, carbon nanotube and graphene [6-9]. To the best of our knowledge, however, all the man-made self-healing materials

\footnotetext{
* Corresponding authors. Tel.: +61 8 92664562; fax: +61 892662681.

E-mail addresses: C.Lu@curtin.edu.au (C. Lu), bhwangqi@sina.com (Q. Wang).
}

reported in the literature require some form of external intervention, such as temperature, pressure and manual fluid injection. Recently, a truly spontaneous self-healing of fractured GaAs nanowires (NWs) was observed in in situ deformation experiments inside a transmission electron microscope under near-vacuum conditions [10]. It has been shown from molecular dynamics simulations that the rebonding of $\mathrm{Ga}$ and As atoms across fracture surfaces plays a key role in restoring these NWs [11].

Since relatively smooth fracture surfaces can be realized in structures with ultrathin lateral dimensions, it seems that self-healing may be an intrinsic property of one-dimensional nanomaterials [11-15]. However, self-healing has rarely been observed in GaAs NWs with lateral dimensions more than $12 \mathrm{~nm}$. Moreover, there is a practical interest in developing materials that can achieve multiple healing cycles [5]. Our recent in situ observations have revealed 
that GaAs NWs possess the ability to heal themselves when subjected to repeated fractures [10]. However, it has been difficult to quantify this healing behavior using experiments. Here we show that these issues can be addressed at least partially with numerical simulations.

In this paper, molecular dynamics simulations are performed to investigate the self-healing behavior of GaAs NWs, focusing on two important issues: (i) how GaAs NWs lose the self-healing capacity as their lateral dimensions increase and (ii) the effect of repeated fracture on the healing efficiency. The effects of other factors, such as temperature, oriented attachment and atomic diffusion, are also investigated.

\section{Computational methods}

An as-synthesized GaAs NW has a zinc-blende structure with a threefold symmetry around the [1 111$]$ axis and (1 110$)$ side facets [16]. Fig. 1a shows a typical transmission electron microscopy image of an individual GaAs NW and Fig. 1b illustrates a typical computational model, generated by visual molecular dynamics [17], with a cross-sectional dimension of $5.5 \mathrm{~nm}$ and an aspect ratio of 6:1. Generally, NWs are made of an ordered sequence of three basic structure modules of tetrahedral bonding [18]. Here, a supercell concept is adopted. Specifically, a vacuum region of $3 \mathrm{~nm}$ is attached on the outside (lateral) surface to ensure traction-free conditions. Periodic boundary conditions are implemented along all directions. To study the size effect, the lateral dimensions of the NWs range from 1.4 to $9.2 \mathrm{~nm}$. Analyses are carried out at temperatures ranging from 300 to $1200 \mathrm{~K}$ to quantify the temperature effect on the self-healing behavior. A typical NW with the lateral dimension of $5.5 \mathrm{~nm}$ is selected to investigate the influence of repeated fracture and healing on its mechanical properties.
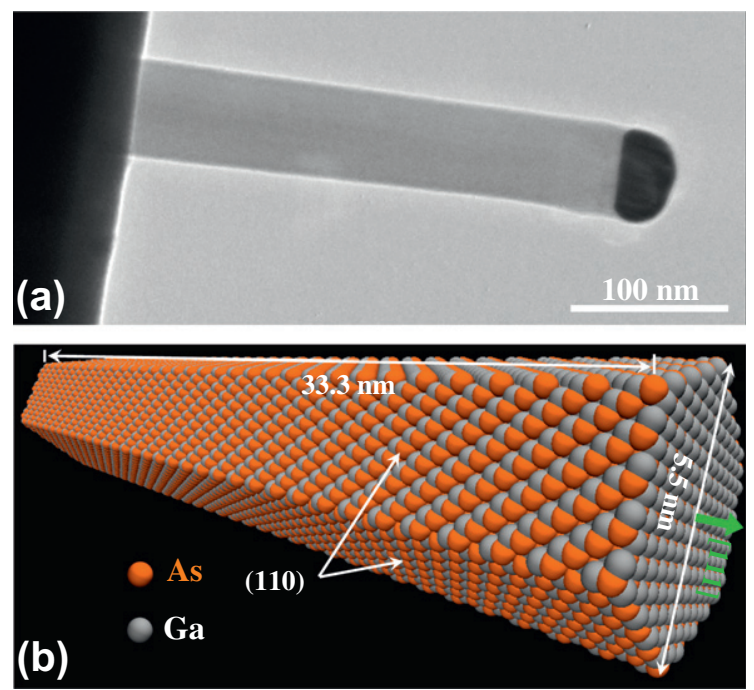

Fig. 1. (a) Transmission electron microscopy image of an individual GaAs NW and (b) the corresponding computational model.
A potential function consisting of two-body $(\mathrm{Ga}-\mathrm{Ga}$, $\mathrm{Ga}-\mathrm{As}$ and As-As) and three-body covalent interactions ( $\mathrm{Ga}-\mathrm{As}-\mathrm{Ga}$ and $\mathrm{As}-\mathrm{Ga}-\mathrm{As}$ ) with the form of

$V=\sum_{i<j} V_{i j}^{(2)}\left(r_{i j}\right)+\sum_{i, j<k} V_{j i k}^{(3)}\left(r_{i j}, r_{i k}\right)$

is used in the molecular dynamics simulations, where $r_{i j}$ is the distance between atoms $i$ and $j$ [19]. The two-body part can be written as

$$
\begin{aligned}
V_{i j}^{(2)}\left(r_{i j}\right)= & A_{i j}\left(\frac{\sigma_{i}+\sigma_{j}}{r_{i j}}\right)^{\eta_{i j}}+\frac{Z_{i} Z_{j}}{r_{i j}} e^{-r_{i j} / r_{1 s}} \\
& -\frac{\alpha_{i} Z_{j}^{2} \alpha_{j} Z_{i}^{2}}{2 r_{i j}^{4}} e^{-r_{i j} / r_{4 s}}-\frac{W_{i j}}{r_{i j}^{6}}
\end{aligned}
$$

The first term represents the steric repulsion, which is described by the strength prefactors $A_{i j}$, ionic radii $\sigma_{i}$ and $\sigma_{j}$, and the exponent of steric repulsion $\eta_{i j}$. The second term is the screened Coulomb interaction due to charge transfer, with $Z_{i}$ and $Z_{j}$ being the effective atomic charges and $r_{1 s}$ the screen length. The third term corresponds to the charge dipole interaction due to the large polarizablity of negative ions, where $r_{4 s}$ is the screen length. The last term is the induced dipole-dipole interaction containing the van der Waals strength $W_{i j}$.

The three-body term in Eq. (1) combines the spatial and angular dependence in the form of

$$
\begin{aligned}
V_{j i k}^{(3)}\left(r_{i j}, r_{i k}\right)= & B_{j i k} \exp \left(\frac{l}{r_{i j}-r_{0}}+\frac{l}{r_{i k}-r_{0}}\right) \times \frac{\left(\cos \theta_{j i k}-\cos \bar{\theta}_{j i k}\right)^{2}}{1+C_{j i k}\left(\cos \theta_{j i k}-\cos \bar{\theta}_{j i k}\right)^{2}} \\
& \times \Theta\left(r_{0}-r_{i j}\right) \Theta\left(r_{0}-r_{i k}\right)
\end{aligned}
$$

where $B_{j i k}=7.9 \times 10^{-19} \mathrm{~J}$ is the strength of interaction, $\theta_{j i k}$ is the angle between $r_{i j}$ and $r_{i k}$, and $C_{j i k}=20$ and $\bar{\theta}_{j i k}=109.47^{\circ}$ are constants. $\Theta\left(r_{0}-r_{i j}\right)$ is a step function. The characteristic length $l$ and the three-body cutoff length $r_{0}$ are set to 0.1 and $0.38 \mathrm{~nm}$, respectively. The two-body interaction is truncated at $r_{c}=0.75 \mathrm{~nm}$. To keep the potential and its first derivative continuous at $r_{c}$, the two-body interaction is shifted with

$$
V_{(i j)}^{(2 \text { shifted })}(r)=\left\{V_{i j}^{(2)}(r)-V_{i j}^{(2)}\left(r_{c}\right)-\left(r-r_{c}\right)\left(d V_{i j}^{(2)}(r) / d r\right)_{r=r_{c}}, \text { if } r \leqslant r_{c}, \text { and } 0 \text { if } r>r_{c}\right.
$$

Parameters of the two-body potential are listed in Table $1[19]$.

To simulate uniaxial tensile loading, a deformation increment along the [1 11 ] direction is applied in two steps: a modified isothermal-isobaric ensemble is first used to stretch a GaAs NW with a strain rate of $0.001 \mathrm{ps}^{-1}$ for $1 \mathrm{ps}[20]$; then the axial strain is held while the NW is relaxed for $6 \mathrm{ps}$ via a canonical ensemble to obtain its mechanical parameters [21]. In each loading step, the nominal strain is applied with a small increment of $0.1 \%$. The attaching operation is accomplished by a similar technique as the tensile loading, but with a negative strain rate $\left(-0.001 \mathrm{ps}^{-1}\right)$. Although the strain rate in the simulations is much higher than that in experiments, the simulated Young's modulus, strength and fracture behavior of NWs are consistent with in situ tests. Since these are the 
Table 1

Two-body potential parameters used in the interaction potential of GaAs NWs, where $r_{1 s}=0.5 \mathrm{~nm}, r_{4 s}=0.375 \mathrm{~nm}$, and $r_{c}=0.75 \mathrm{~nm}[19]$ (see Eqs. (1)(4)).

\begin{tabular}{lllccc}
\hline Atom type & $\sigma(\mathrm{nm})$ & $\alpha\left(\mathrm{nm}^{3}\right)$ & $Z_{i}(e)$ & $A_{i j}\left(10^{-19} \mathrm{~J}\right)$ & $\eta_{i j}$ \\
\hline $\mathrm{Ga}$ & 0.095 & 0 & 0.9418 & & \\
$\mathrm{As}$ & 0.1498 & 0.002 & -0.9418 & & \\
$\mathrm{Ga}-\mathrm{Ga}$ & & & 16.4984 & 7 & 0 \\
$\mathrm{Ga}-$ As & & & 2.0623 & 9 & 58.916 \\
As-As & & & 2.0623 & 7 & 0 \\
\hline
\end{tabular}

$e$ represents the electronic charge.

most critical properties in the healing process under study, we believe that the atomistic simulations are suitable for the present study. All samples are relaxed for $20 \mathrm{ps}$ before stretching or attachment. The stress tensor is calculated by a modified virial formula [22]. More discussion on numerical simulations can be found in Ref. [23]. All the calculations are carried out using the DL_POLY2.20 package [21].

It is shown that, due to the higher stress state in the surface area of NWs [24-27], the Young's modulus of NWs varies with their lateral dimensions. This phenomenon can be described by the employed interatomic potential. The predicted size-dependent Young's modulus of GaAs NWs is consistent with recent in situ compression tests [28]. Surface properties of GaAs for the (100), (110) and (111) surfaces predicted by the present interatomic potential are also in agreement with ab initio calculations and experimental measurements, such as the (100) surface reconstruction [19]. These factors are of significance in characterizing the interaction between two fractured free surfaces, where self-healing occurs along (1111) planes.

\section{Results}

\subsection{Self-healing process}

The self-healing behavior of a fractured GaAs NW is assessed in three steps: loading, self-healing and reloading. First, a uniaxial tensile load is applied on a GaAs NW until it fractures. Then the two fractured sections are attached along the common axis. Finally, a uniaxial tensile load is reapplied and the mechanical properties of the restored NW are evaluated.

As shown in Fig. 2a, a typical self-healing process consists of three stages: attaching, contacting and healing. (i) When the distance between two fracture surfaces is larger than $1.0 \mathrm{~nm}$, there is no detectable interaction. (ii) As the distance reduces from 1.0 to $0.7 \mathrm{~nm}$, substantial surface attraction emerges. The attractive force per unit area is about $0.1 \mathrm{GPa}$, which enables the rebuilding of a small amount of broken $\mathrm{Ga}-\mathrm{As}$ bonds between two fracture surfaces. (iii) With further reduction in distance, the surface attraction force per unit area between two fracture surfaces reaches a maximum of $0.87 \mathrm{GPa}$, which is much stronger than that in the contacting stage. The two peaks of surface attraction in Fig. 2a correspond to two typical healing events: partial healing in rough regions (peak A) and full
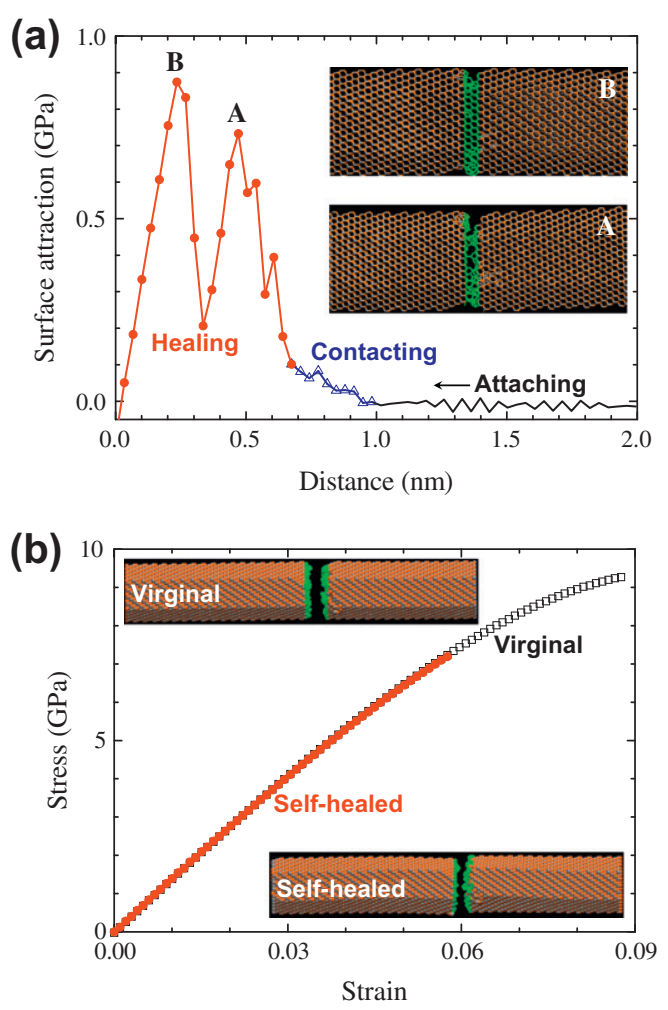

Fig. 2. Self-healing of a fractured GaAs NW. (a) The three-stage healing process: attaching, contacting and healing. Insets show patterns at two peaks (A and B) in the healing stage. (b) Stress vs. strain curves of a virgin and a self-healed GaAs NW.

healing of the fractured NW (peak B). The tensile test of a self-healed GaAs NW shows that the tensile strength can reach $7.19 \mathrm{GPa}$. In comparison with its original strength of $9.27 \mathrm{GPa}$, the healing efficiency is $78 \%$ (see Fig. 2b). However, the Young's modulus (138.0 GPa) of the self-healed GaAs NW is almost the same as that of the virgin sample (138.7 GPa). Here, it is of interest to note that the second brittle failure happens at the same location. For a detailed description of the self-healing process, the reader is referred to Ref. [11].

\subsection{Size effect}

In situ tensile tests on pristine GaAs NWs have shown that their mechanical behaviors are strongly size dependent. When their cross-sectional dimensions exceed a 
critical size (around $2.3 \mathrm{~nm}$ ), GaAs NWs are predominantly brittle; otherwise, they exhibit considerable plastic deformation (see Fig. 3a). As the lateral dimension increases from 1.4 to $9.2 \mathrm{~nm}$, the Young's modulus reduces by $6 \%$, from 147.5 to $138.1 \mathrm{GPa}$, while the tensile strength does not exhibit a clear monotonic trend (see Fig. 3b). GaAs NWs with lateral dimensions less than $2.3 \mathrm{~nm}$ (e.g. the cases of 1.4 and $1.9 \mathrm{~nm}$ in Fig. 3) possess lower tensile strengths. As the dimension increases from 2.3 to $9.2 \mathrm{~nm}$, the tensile strength decreases by $1 \%$, from 9.33 to 9.24 GPa. It is seen from Fig. 3a that the NW with a lateral dimension of $1.4 \mathrm{~nm}$ exhibits the biggest elongation of $30 \%$, in contrast to that of $\sim 9 \%$ for brittle failure. In this extreme case, the plastic elongation leads to the formation of a single atom chain, similar to the behavior of ultrathin metal NWs [29] (see the insets in Fig. 3a).

Fig. 4a illustrates five adjacent $\mathrm{Ga}-\mathrm{As}$ layers, each layer representing one module of the tetrahedral bonding [18]. The brittle fracture initiates through the breaking of $\mathrm{Ga}-$ As bonds at the middle layer, which subsequently develops into two fracture surfaces (see Fig. 4b). The breaking of bonds also occurs in neighboring layers, and may lead to the formation of stripping flaps, as shown in Fig. 4c. These stripping flaps eventually deposit on the fracture surfaces and result in surface roughness. The larger the lateral dimension of a GaAs NW, the stronger this feature is.
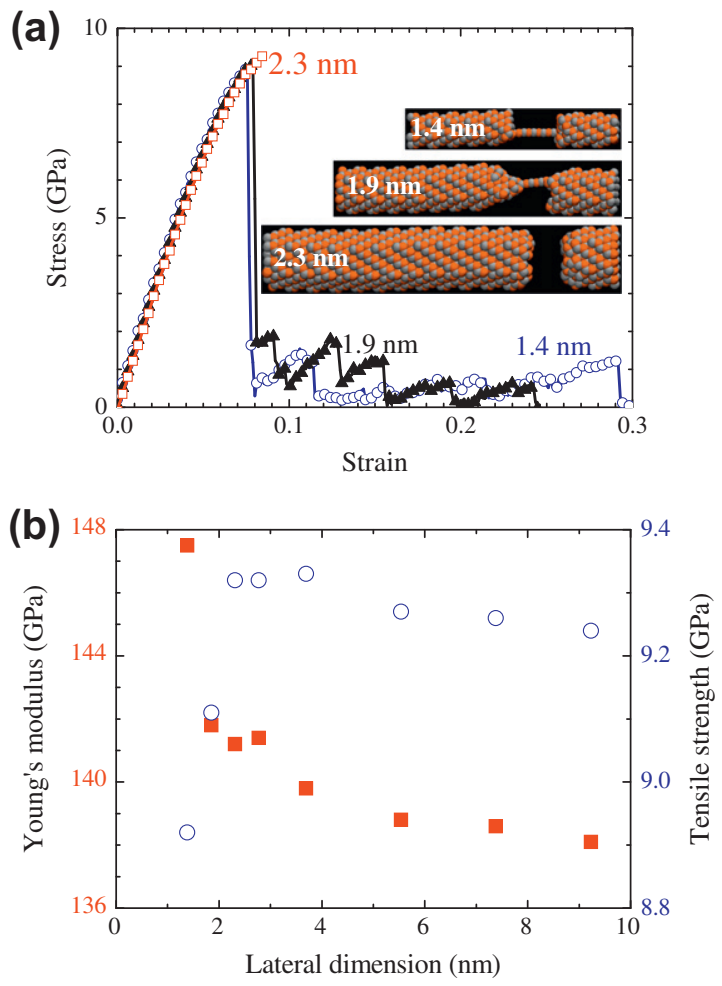

Fig. 3. Size-dependent mechanical behaviors of GaAs NWs. (a) Plastic behavior is seen as the lateral dimension of NWs is less than $2.3 \mathrm{~nm}$, while brittle failure occurs in NWs with larger lateral dimensions. Insets show typical patterns of plastic deformation and brittle fracture. Young's modulus and tensile strength are plotted in (b) as functions of the lateral dimension.
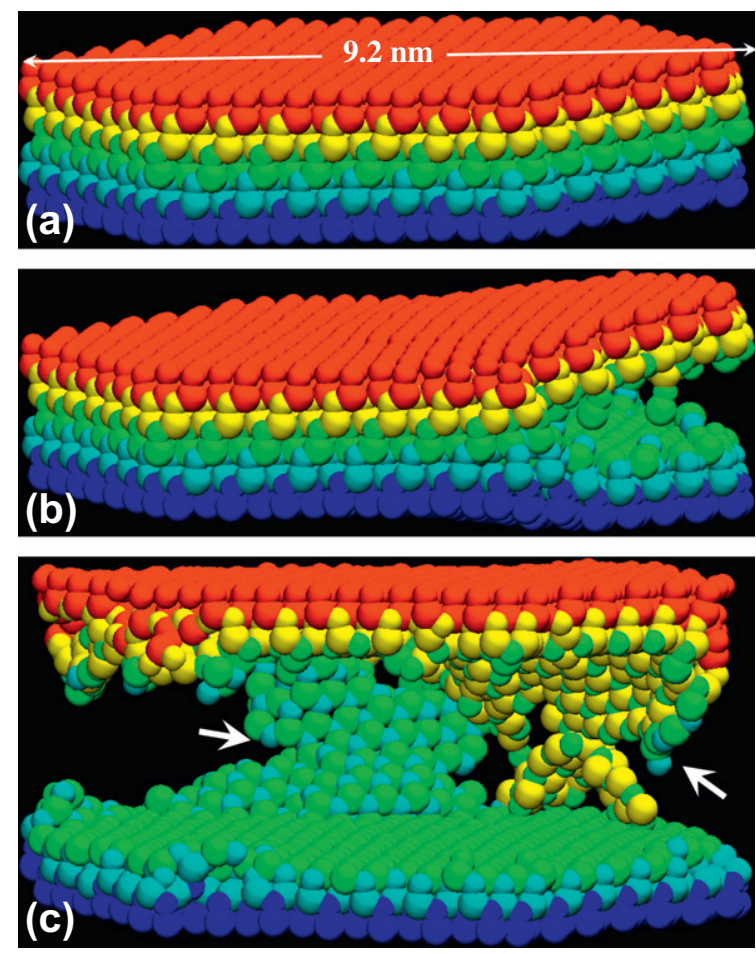

Fig. 4. Brittle fracture of a GaAs NW with a lateral dimension of $9.2 \mathrm{~nm}$. (a) Five adjacent $\mathrm{Ga}-\mathrm{As}$ layers. Bigger and smaller spheres represent $\mathrm{Ga}$ and As, respectively. Atoms on each layer are denoted by the same color. (b) Brittle fracture occurs through breaking of $\mathrm{Ga}-\mathrm{As}$ bonds at the middle layer. (c) $\mathrm{Ga}-\mathrm{As}$ bonds breaking at neighboring layers lead to the formation of stripping flaps as two fractured sections separate (indicated by arrows).

The joining of materials without heating is usually referred to as cold welding [30]. Here, our attention is focused on the self-healing of GaAs NWs after brittle fracture. As shown in Fig. 5a, the roughness of fracture surfaces increases with increasing lateral dimension of a GaAs NW. When the lateral dimension is less than $5.5 \mathrm{~nm}$, its roughness is only $1-2$ monolayers thick. However, for an NW with a lateral dimension of $9.2 \mathrm{~nm}$, the thickness of its rough region reaches about $2 \mathrm{~nm}$. The measured surface attraction (defined as the maximum attractive traction during the healing stage) decreases by $65 \%$, from 1.74 to $0.61 \mathrm{GPa}$, as the lateral dimension increases from 2.3 to $9.2 \mathrm{~nm}$. As a result, the healing efficiency reduces by $45 \%$, from 84 to $46 \%$ (see Fig. 5 b).

\subsection{Repeated fracture}

Based on simulations of repeated fracture and healing of GaAs NWs, the restored tensile strength reduces by $64 \%$, from 7.19 to $2.56 \mathrm{GPa}$, in 24 healing cycles. The restored tensile strength drops continuously during the first 24 cycles, then remains stable at around $2.50 \mathrm{GPa}$, which accounts for $27 \%$ of the virgin strength $(9.27 \mathrm{GPa}$; see Fig. 6a). Repeated fracture also causes a reduction in the restored Young's modulus. Specifically, during the first 22 healing cycles, the restored Young's modulus decreases 
(a)

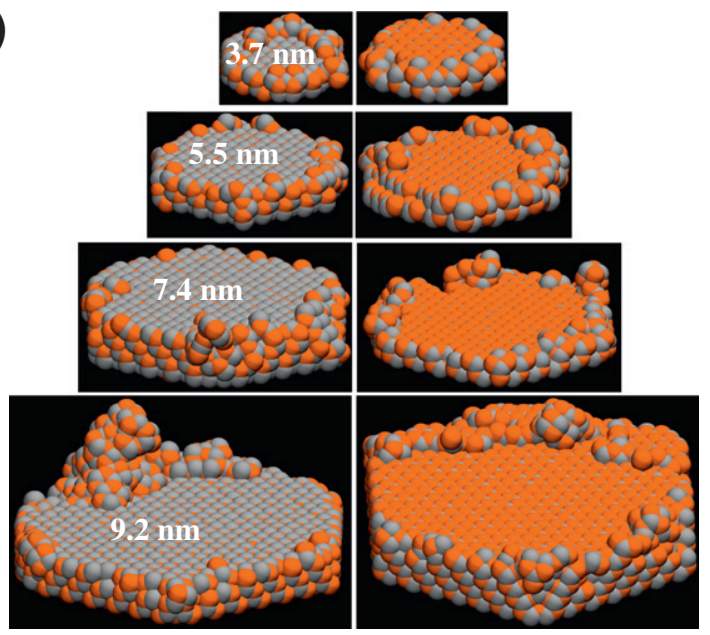

(b)

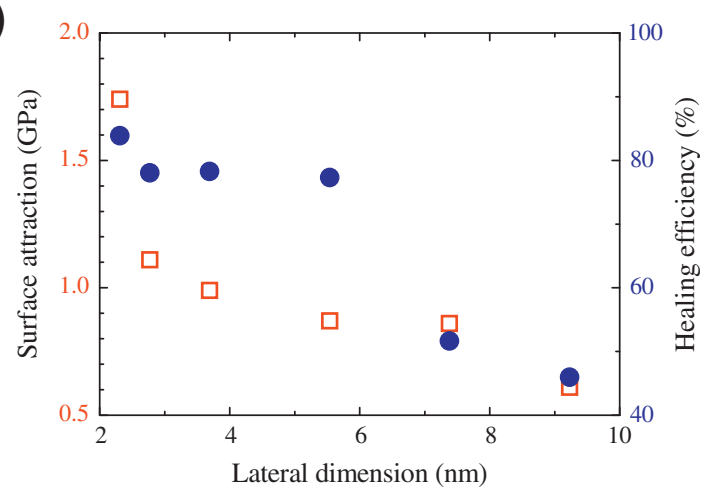

Fig. 5. Size-dependent self-healing behavior of a fractured GaAs NW. (a) Fracture morphologies and (b) surface attraction and healing efficiency vs. the lateral dimension, respectively.

by $4 \%$, from 138.0 to $132.1 \mathrm{GPa}$. After that, the Young's modulus fluctuates in a range of $7 \%$ around $120.0 \mathrm{GPa}$, as shown in Fig. 6b. Also, repeated fracture intensifies the roughness of the fracture surfaces due to the mismatch of two fractured sections during the attaching operation; see the surface pattern at the eleventh healing cycle in Fig. 7.

\subsection{Temperature effect}

As the temperature increases from 300 to $1200 \mathrm{~K}$, the healing efficiency fluctuates between $60 \%$ (at $1000 \mathrm{~K}$ ) and $81 \%$ (at $700 \mathrm{~K}$ ). Similarly, the restored Young's modulus fluctuates from 136.7 to $138.2 \mathrm{GPa}$, as shown in Fig. 8a. Compared to the Young's modulus of $138.7 \mathrm{GPa}$ for the virgin sample, at least $99 \%$ restoration has been achieved. The surface attraction undergoes a wide fluctuation, from 0.81 to $1.21 \mathrm{GPa}$. The strongest surface attraction occurs at $700 \mathrm{~K}$, which coincides with the best healing efficiency. The worst healing efficiency, at $1000 \mathrm{~K}$, corresponds to a case of weak surface attraction (see Fig. 8b). It is worth noting, however, that the influence of temperature analyzed here only addresses instant $\mathrm{Ga}-\mathrm{As}$ rebonding without the contribution of atomic diffusion.
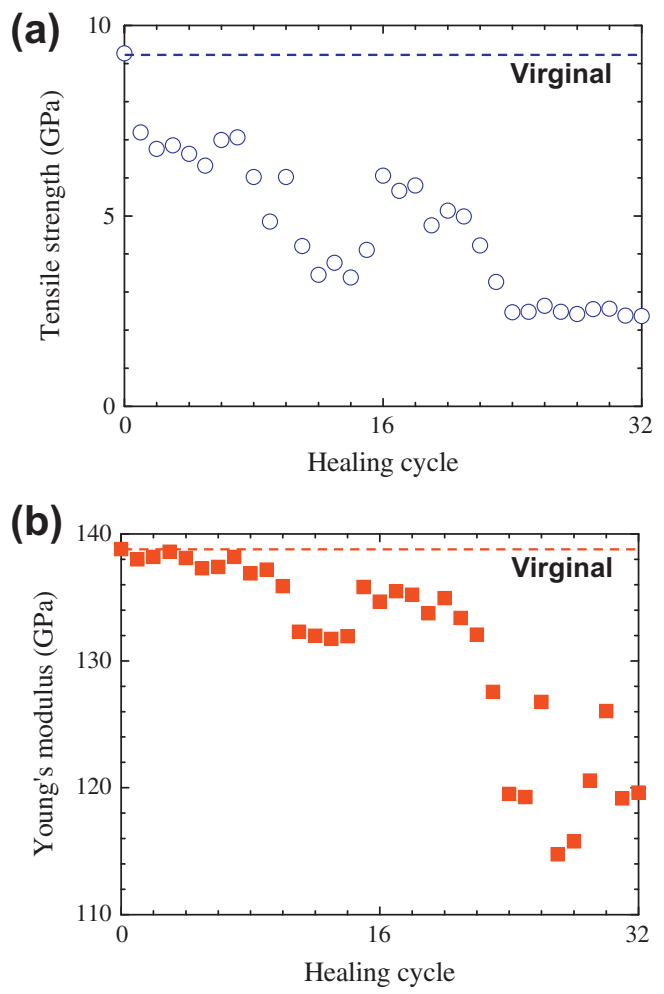

Fig. 6. Effect of repeated fracture and cure in a fractured GaAs NW. (a) and (b) show that the restored tensile strength and Young's modulus vary with healing cycles. Dashed lines are their virgin values of 9.27 and 138.7 GPa, respectively.

\subsection{Atomic diffusion}

To accelerate atomic diffusion, the GaAs NW subjected to 31 healing cycles is annealed at $1400 \mathrm{~K}$, with the annealing time varying from 0.1 to $5 \mathrm{~ns}$. The results show that, with an annealing treatment of $0.1 \mathrm{~ns}$, the healing efficiency is substantially improved, from 26 to $68 \%$, as shown in Fig. 9. When the annealing time is beyond $1 \mathrm{~ns}$, the healing efficiency fluctuates between 77 and $84 \%$, with an average value of $80 \%$. Patterns at the healing sections indicate that atoms near the fracture sites spontaneously diffuse to fill gaps between two surfaces and restore the original [1111]orientated lattice structure. However, due to the rather rough fracture morphology generated after multiple healing cycles, atoms on the fracture surfaces cannot completely return to their original (110) orientations. Instead, small (111) plane reconstructs are observed at local regions (see the insets of Fig. 9).

\section{Discussion}

\subsection{Factors that facilitate self-healing}

Surface attraction appears to be the key factor in a self healing process. When a GaAs NW is subjected to tensile loading, fracture mainly occurs between two neighboring (111) planes. The relatively smooth fracture surfaces due 
Cycle

1

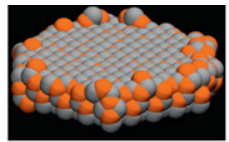

11

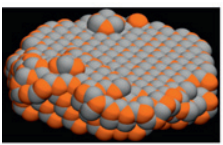

21
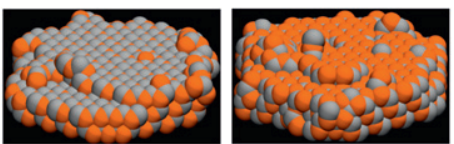

31
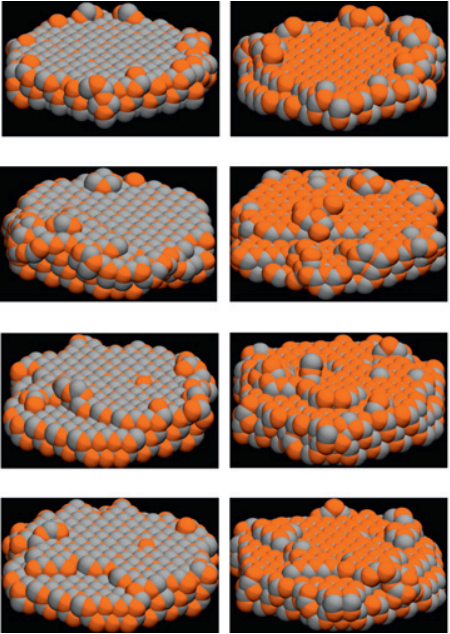

Self-healed pattern
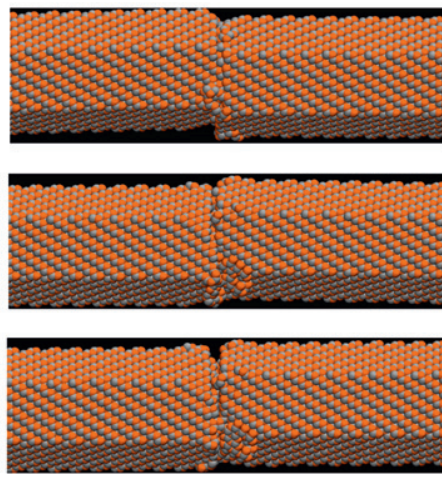

Fig. 7. Fracture morphologies and self-healed patterns at different healing cycles.
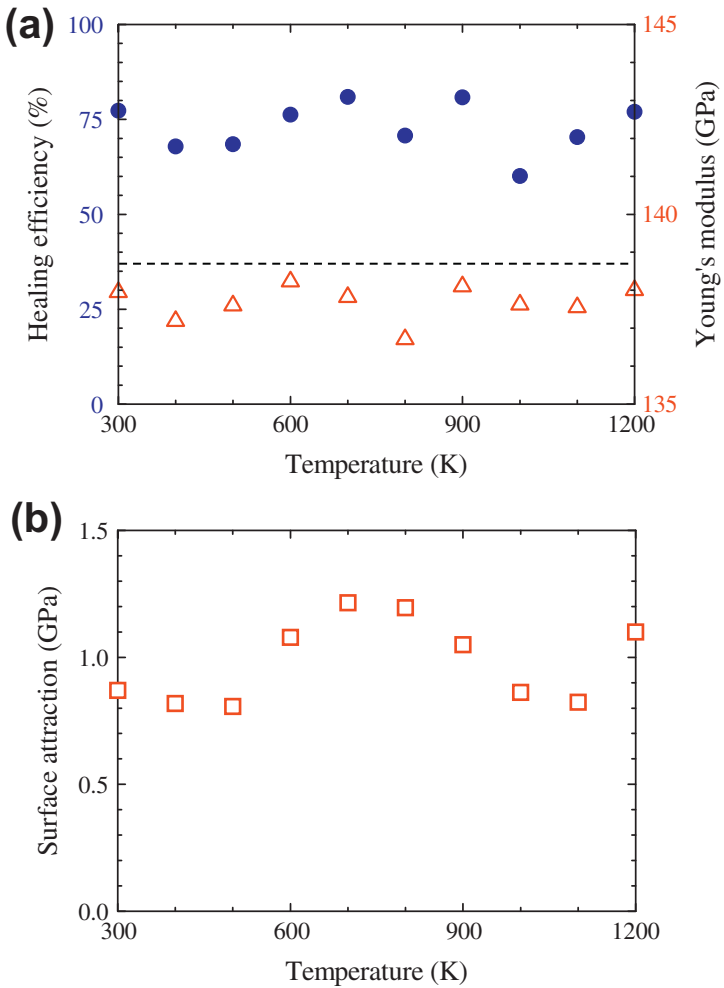

Fig. 8. Influence of temperature on the self-healing behavior of a GaAs NW with a lateral dimension of $5.5 \mathrm{~nm}$. (a) The healing efficiency and restored Young's modulus vs. temperature, where dashed line indicates the virgin Young's modulus of $138.7 \mathrm{GPa}$; (b) the corresponding surface attraction.

to its ultrathin lateral dimension provide a precondition for self-healing. Here, two opposite fracture surfaces are occupied by oppositely charged $\mathrm{Ga}$ and As atoms. As the distance between two fracture surfaces falls below $1.0 \mathrm{~nm}$, surface attraction emerges as a result of the near-field electrostatic interaction. At a long distance, the Coulomb

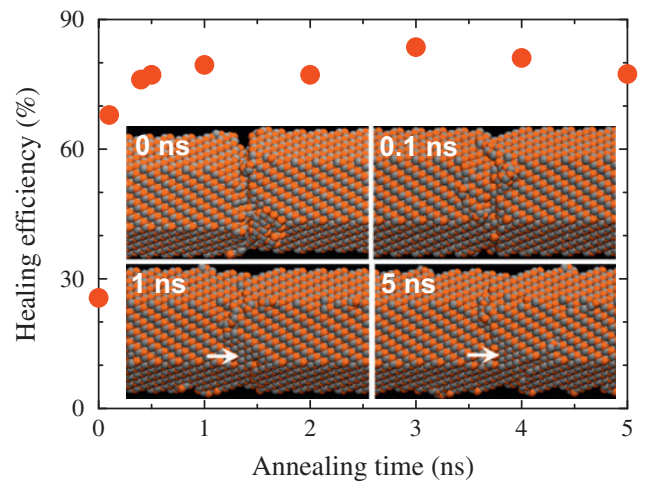

Fig. 9. Healing efficiency vs. annealing time. The insets show snapshots at different annealing times under $1400 \mathrm{~K}$. The arrows indicate a small reconstructed (111) surface at the healing site.

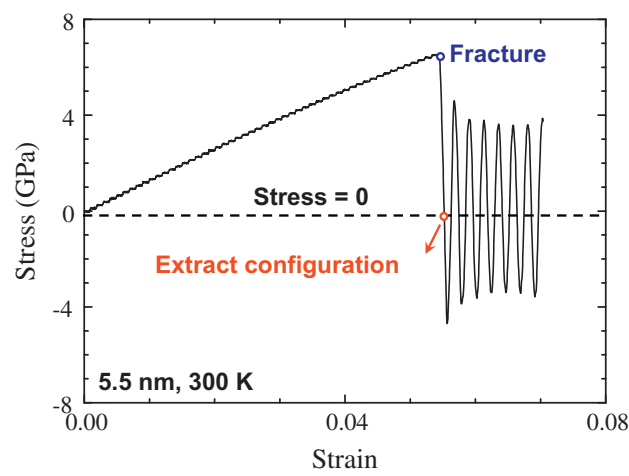

Fig. 10. Removal of vibrational energy. Stress and strain data are taken from tensile tests after the third healing cycle.

interaction between two fracture surfaces quickly declines due to the alternate arrangement of oppositely charged $\mathrm{Ga}$ and As ions. At smaller distances, surface attraction can be strong enough to pull those atoms located at opposite fracture surfaces together and induce $\mathrm{Ga}-\mathrm{As}$ 
rebonding. The original structure of a GaAs NW can be mostly restored via large-scale rebonding at fractured sites. A similar role of surface attraction was also discovered in $\mathrm{ZnO}$ and gold nanocrystallites [30-32], except that, due to the non-polar nature of gold atoms, forces such as the van der Waals interaction are likely to play a more important role. As the lateral dimension of GaAs NWs increases, the dramatically reduced surface attraction leads to poor healing efficiency. This explains why self-healing behavior is rarely observed in GaAs NWs with lateral dimensions exceeding $12 \mathrm{~nm}[10]$.

An oriented attachment operation can be introduced to facilitate the self-healing of fractured GaAs NWs. Such an operation ensures that atoms and $\mathrm{Ga}-\mathrm{As}$ bonds can return to their original positions when rebonding happens. For example, healing always occurred instantaneously between two gold NWs with the same growth orientation [30]. The effect of oriented attachment is reflected by a deviation in the healing efficiency from the attenuation trend during the first 24 repeated healing cycles (see Fig. 6a). Wellaligned fractured sections along the [1 111 ] orientation produces better efficiency, and vice versa. A similar mechanism was reported in PdSe nanocrystals [33,34].

Atomic diffusion can be helpful in further removing the mismatched $\mathrm{Ga}-\mathrm{As}$ bonds at healing sites and moving atoms to their original [111]-oriented lattice structure. Such a rearrangement via rebonding experiences an energy reduction by reducing the surface area. It is worth noting, however, that the diffusion of atoms also introduces intrinsic irregularities on fracture surfaces. As the sample is annealed beyond $1 \mathrm{~ns}$, atomic diffusion reaches a dynamical equilibrium, which causes a slight fluctuation in healing efficiency around $80 \%$, with the amplitude of $3 \%$. Rebonding facilitated by atomic diffusion has been reported in most nanomaterials [35-37].

\subsection{Factors that hinder self-healing}

Several factors, such as fracture surface roughness, impurities, local defects and thermal vibration, can be obstacles to the self-healing of a fractured GaAs NW. Although typical rough sections on fracture surfaces are only several atoms thick, they can prevent more atoms from entering the effective range of surface attraction. Roughness also causes the mismatch of atoms during the healing process. Thus, the original structure of an NW cannot be entirely restored. The local defects left in a selfhealed GaAs NW become the source of damage nucleation during reloading. As a result, the self-healed GaAs NW shows a lower Young's modulus and tensile strength. The restored Young's modulus and healing efficiency decrease as the healing cycles increase. Due to the ultrathin size, roughness caused by repeated fracture reaches a stable state after 24 healing cycles. The number of rebuilt GaAs bonds remains constant during subsequent healing cycles. This leads to an approximately constant tensile strength, which is dependent on the total number of restored $\mathrm{Ga}-\mathrm{As}$ bonds. However, the random mismatch between atoms occurring in isolated healing events results in a fluctuation in the Young's modulus. The negative influence of surface roughness on self-healing has also been recognized in polymers [38-40]. Furthermore, the healing efficiency fluctuates as the attaching process is operated at diverse temperatures, which is due to the stochastic $\mathrm{Ga}-\mathrm{As}$ rebonding between fractured sites caused by the thermal motion of surface atoms and unstable oriented attachment operation.

\section{Conclusions}

Based on molecular dynamics simulations, we have shown that the self-healing behavior of fractured GaAs NWs depends on their lateral dimensions and the number of healing cycles. Sufficiently smooth fracture surfaces and strong surface attraction appear to be preconditions for self-healing of fractured GaAs NWs. The surface attraction due to the electrostatic interaction in the nearfield region contributes to the $\mathrm{Ga}-\mathrm{As}$ rebonding. However, a large lateral dimension and repeated fracture increase the roughness of the fractured surfaces and hinder the selfhealing of GaAs NWs. As the lateral dimension of a GaAs NW increases from 2.3 to $9.2 \mathrm{~nm}, 65 \%$ of surface attraction is lost and the healing efficiency reduces by $46 \%$. For NWs with a lateral dimension of $5.5 \mathrm{~nm}$ at $300 \mathrm{~K}$, the restored tensile strength decreases by $64 \%$ after 24 healing cycles, then saturates to a healing efficiency of $27 \%$. The thermal motion of surface atoms increases the mismatch of atoms between two fracture surfaces during an attaching operation, which causes fluctuations in the healing efficiency. Atomic diffusion induced by an annealing treatment helps to eliminate the mismatch and enhances the healing efficiency. The present study provides useful insights into the self-healing mechanisms of GaAs NWs and suggests that more novel nanostructured materials, such as $\mathrm{SiC}, \mathrm{ZnO}$ and $\mathrm{GaN}$, can be designed with the ability to self-heal upon fracture.

\section{Acknowledgements}

This work has been supported by the Australian Research Council (Grant Nos. DP0985450 and FT110100236), the National Natural Science Foundation of China (Grant Nos. 11172024, 11172305, 10932011, 11072014, 10972218 and 11021262), the National Basic Research Program of China (2012CB937500), the China Postdoctoral Science Foundation (2011M500211), the Opening Fund of State Key Laboratory of Nonlinear Mechanics, the Research Grant Council of the Hong Kong Special Administrative Region, China (Project No. 9041679 (CityU 120611)) and the US National Science Foundation (CMMI-0758535). Computations were performed on the LNM blade, computer clusters at the CNIC supercomputing Centre and iVEC through the use of advanced computing resources located at iVEC@ARRC. Partially 
computational resources were provided by the Intersect Australia Ltd.

\section{Appendix A. How to remove the vibrational energy}

In ideally brittle materials, there is no energy dissipation mechanism during external loading or after brittle fracture. All the external energy input is stored as elastic energy during external loading. After fracture, the elastic energy transfers to vibration energy, which can be dissipated by heat exchange between the specimen and the environment within several seconds or minutes. During vibration, the internal energy $E$ of a system can be written as

$E=E_{k}+E_{p}$

where $E_{\mathrm{k}}$ and $E_{\mathrm{p}}$ are the kinetic energy and the potential energy, respectively. The minimum value of $E_{\mathrm{p}}$ corresponds to a zero stress state, a vibrational system rebounding to its original shape. In molecular dynamics simulations, it is impractical to dissipate such a real physical vibration by thermostat. Therefore, we adopt the following approach to remove the vibration. First, we intensify data records around the expected fracture strain. As stress reduces to 0 after fracture, we extract the corresponding configuration and remove atoms' velocities, as shown in Fig. 10. Thereafter the sample is relaxed at a desired temperature before a further attaching operation is applied.

\section{References}

[1] White SR, Sottos NR, Geubelle PH, Moore JS, Kessler MR, Sriam SR, et al. Nature 2001;409:794.

[2] Cordier P, Tournilhac F, Ziakovic CS, Leibler L. Nature 2008;451:977.

[3] Mynar JL, Aida T. Nature 2008;451:895.

[4] Chen X, Dam MA, Ono K, Mal A, Shen H, Nutt SR, et al. Science 2002;295:1698.

[5] Toohey KS, Sottos NR, Lewis JA, Moore JS, White SR. Nat Mater 2007;6:581.

[6] Puzder A, Williamson AJ, Gygi F, Galli G. Phys Rev Lett 2004;92:217401.

[7] Börrnert F, Gorantla S, Bachmatiuk A, Warner JH, Ibrahim I, Thomas J, et al. Phys Rev B 2010;81:201401.

[8] Bläckberg L, Ringbom A, Sjöstrand H, Klintenberg M. Phys Rev B 2010;82:195434.
[9] Amendola V, Meneghetti M. Nanoscale 2009;1:74.

[10] Wang YB, Joyce HJ, Gao Q, Liao XZ, Tan HH, Zou J, et al. Nano Lett 2011;11:1546.

[11] Wang J, Lu C, Wang Q, Xiao P, Ke FJ, Bai YL, et al. Europhys Lett 2012;98:16010.

[12] Agrawal R, Paci JT, Espinosa HD. Nano Lett 2010;10:3432.

[13] Agrawal R, Espinosa HD. Nano Lett 2011;11:786.

[14] Wang J, Lu C, Wang Q, Xiao P, Ke FJ, Bai YL, et al. Europhys Lett 2011;95:63003

[15] Wang J, Lu C, Wang Q, Xiao P, Ke FJ, Bai YL, et al. Nanotechnology 2012;23:025703.

[16] Sköld N, Wagner JB, Karlsson G, Hernan T, Seifert W, Pistol M, et al. Nano Lett 2006;6:2743.

[17] Humphrey W, Dalke A, Schulten K. J Mol Graph 1996;14:33.

[18] Lindefelt $U$, Iwata $H$, Öberg S, Briddon PR. Phys Rev B 2003;67:155204.

[19] Su X, Kalia RK, Nakano A, Vashishta P, Madhukar A. J Appl Phys 2003;94:6762.

[20] Melchionna S, Ciccotti G, Holian BL. Mol Phys 1993;78:533.

[21] Smith W, Yong CW, Rodger PM. Mol Simul 2002;28:385.

[22] Zhou M. Proc Roy Soc London Ser A 2003;459:2347.

[23] Wang J, Kulkarni AJ, Ke FJ, Bai YL, Zhou M. Comput Methods Appl Mech Eng 2008;197:3182, and references therein.

[24] Chen CQ, Shi Y, Zhang YS, Zhu J, Yan YJ. Phys Rev Lett 2006;96:075505.

[25] Agrawal R, Peng B, Gdoutos EE, Espinosa HD. Nono Lett 2008;8:3668.

[26] Xiao P, Wang X, Wang J, Ke FJ, Zhou M, Bai YL. Appl Phys Lett 2009;95:211907.

[27] Xiao P, Wang J, Ke FJ, Bai YL. Compos Part B - Eng 2012;43:57.

[28] Wang YB, Wang LF, Joyce HJ, Gao Q, Liao XZ, Mai YW, et al. Adv Mater 2011;23:1356.

[29] da Silva EZ, da Silva Antônio JR, Fazzio A. Phys Rev Lett 2001;87:256102.

[30] Lu Y, Huang JY, Wang C, Sun SH, Lou J. Nat Nanotechnol 2010;5:218.

[31] Kizuka T, Yamada K, Tanaka N. Appl Phys Lett 1997;70:964.

[32] Kizuka T, Tanaka N. Philos Mag Lett 1994;69:135.

[33] Cho KS, Talapin DV, Gaschler W, Murray CBJ. J Am Chem Soc 2005; 127:7140.

[34] Van Huis MA, Kunneman LT, Overgaag K, Xu Q, Pandraud G, Zandbergen HW, et al. Nano Lett 2008;8:3959.

[35] Kessler MR, Sottos NR, White SR. Compos Part A - Appl Sci 2003;34:743

[36] Wool RP. Soft Matter 2008;4:400.

[37] Korouš J, Chu MC, Nakatani M, Ando F. J Am Ceram Soc 2000;83:2788.

[38] Kim HJ, Lee KJ, Lee HH. Polymer 1996;37:4593.

[39] Jud K, Kausch HH, Williams JG. J Mater Sci 1981;16:204.

[40] Wu DY, Meure S, Solomon D. Prog Polym Sci 2008;33:479. 